\title{
Effective strategies in recruitment and clinical orientation programme to manage NHS junior doctor workforce shortfall: a district general hospital experience
}

\author{
Authors: Syed Quadery, ${ }^{\mathrm{A}}$ Hamid Roodbari, ${ }^{\mathrm{B}}$ Pradeep Pardeshi, ${ }^{\mathrm{A}}$ Dilip Shah ${ }^{\mathrm{A}}$ and Simon Winn ${ }^{\mathrm{A}}$
}

Table 1. Methodology of behavioural changes

\section{Contexts}

Shortage of junior doctors resulting in increased recruitment of temporary staff at premium rates and inconsistent clinical care

Poor performance and low level of wellbeing in the initial stages at the workplace among new entrant international medical graduates

Risks to patient safety due to poor knowledge of contextual factors including policies and procedures by the new entrant international medical graduates

Managers uncertainty about recruitment and orientation of new entrant international medical graduates

Lack of standardised communication channels between junior doctors, their supervisors and the trust management

Availability of on-site staff accommodation in the trust

Lack of criteria for assessment and unrealistic expectations from new entrant international medical graduates during their orientation

\section{Mechanisms}

Employing new entrant international medical graduates at standard NHS salary

Structured intervention during the orientation period including a good learning environment, adequate mentoring and pastoral support

Providing clear communication of curriculum competencies along with peer support to these doctors

Holding meetings with managers to discuss the design and implementation of the intervention

Developing a standardised communication strategy will facilitate effective communication of critical success factors

Providing free accommodation to doctors doing honorary clinical attachment

Tailoring the intervention based on requirements of junior doctors, their supervisors, and managers of the trust

\section{Outcomes}

A stable workforce leading to improved clinical care and financial position of the trust

Improvement in the participation, selfefficacy and learning of these doctors

Improvement in the awareness among these doctors of the contextual factors contributing to the provision of highquality patient care

Increased participation and support of managers for the intervention

The stakeholders perceive that their voice is being heard and they are valued resulting in their increased satisfaction

Improved cost efficiency of the trust by attracting overseas junior doctors into honorary posts, increasing their recruitment

Clarity of roles and requirements and better participation of the stakeholders

\section{Introduction}

There are significant vacancies of qualified medical professionals across the UK. This has partially been filled by locum doctors causing a major financial burden on the NHS. ${ }^{2}$ To address workforce shortages, the British government has proposed to open five new medical schools to expand national intake by $25 \%{ }^{3}$ The step rise in medical workforce is unlikely to be felt before $2025 .^{3}$

Authors: ${ }^{\text {AEpsom }}$ and St Helier University Hospitals NHS Trust, Epsom, UK; ${ }^{B}$ University Of Sheffield, Sheffield, UK
International medical graduates (IMGs) currently contribute significantly towards the NHS care provision. ${ }^{4}$ Recently there has been an increase in the number of IMGs applying to take the UK medical licensing examinations. ${ }^{5}$ Recruitment of more new entrant IMGs may be the short- to medium-term solution to the junior doctor shortfall. However, IMGs seeking first-time employment in the NHS face multiple challenges. ${ }^{6}$ A robust orientation programme would anticipate and mitigate such challenges and facilitate smooth transition into productive working in the NHS. ${ }^{7}$ There is no standardised orientation programme available for IMGs working in departments of general internal medicine (GIM) in the NHS. 


\section{Methods}

We performed quality improvement interventions of recruitment and a clinical orientation programme (COP) for new entrant IMGs in our organisation employed between December 2017 and April 2019 and developed a framework to anticipate outcomes of these interventions using the realist evaluation methodology.

\section{Results}

Twenty-three IMGs were recruited, $96 \%$ successfully completed the COP with a mean contract duration of $13 \pm 5$ months. To date, $83 \%$ of eligible IMGs have successfully completed their formal annual appraisal. Over the intervention period from academic year $2017 / 2018$ to $2018 / 19$, the mean junior doctor position occupancy has risen from $54 \pm 3$ junior doctors to $73 \pm 4(p<0.001)$. There has been a $€ 1.9$ million reduction in agency and locum junior doctor spend in the division. Exception reporting by trainee junior doctors has fallen by $56 \%$. Formal complaints from patients and their families have fallen by $11 \%$. Length of stay has reduced from $9.3 \pm 16.4$ days to $8.9 \pm 15.6(p=0.035)$ over the same intervention study period. For the first time since its inception, the Care Quality Commission has rated our organisation including the medicine division as 'Good' (from 'Requires Improvement') during the academic year 2018/2019. Context-mechanisms-outcomes configurations detailing the methodology of the behavioural changes implicit to the quality improvement work are shown in Table 1.

\section{Conclusion}

Our recruitment strategy and structured COP provides a stable, trained, and financially sustainable junior doctor workforce. Application in broader NHS settings is recommended.

\section{Conflicts of interest}

None declared.

\section{References}

1 Hughes D, Clarke V. Thousands of NHS nursing and doctor posts lie vacant. BBC News 2016. www.bbc.co.uk/news/health-35667939 [Accessed 21 May 2019].

2 Moberly T. Number of locums has doubled since 2009. BMJ 2016;355:i6207.

3 Al-Attar M. 5 new medical schools to open in England. The Medical Portal. www.themedicportal.com/blog/five-new-medical-schoolsopen-england [Accessed 16 May 2019].

4 British Medical Association. The contribution of international medical graduate doctors to the NHS. BMA, 2015 [Accessed 20 May 2019].

5 General Medical Council. The state of medical education and practice in the UK. GMC, 2018. www.gmc-uk.org/somep2018 [Accessed 08 May 2019].

6 Bhat M, Ajaz A, Zaman N. Difficulties for international medical graduates working in the NHS. BMJ 2014:348:g3120.

7 Hashim A. Educational challenges faced by international medical graduates in the UK. Adv Med Educ Pract 2017;8:441-5. 